\title{
Influência da lidocaína na proteção miocárdica com solução cardioplégica sangüínea
}

\author{
Ricardo Ribeiro DIAS*, Moise DALVA*, Benedito SANTOS*, Karina Lacava KWASNICKA**, Ana Paula \\ SARRAFF**, Altamiro Ribeiro DIAS*, Luis Felipe Pinho MOREIRA*, Noedir Antônio Groppo STOLF*, Sérgio \\ Almeida OLIVEIRA*.
}

* Do Instituto do Coração do Hospital das Clínicas da Faculdade de Medicina da Universidade de São Paulo. **Da Faculdade de Veterinária e Zootecnia da Universidade de São Paulo.

RBCCV 44205-592

\section{Resumo}

Objetivo: Avaliar a eficácia da proteção miocárdica obtida com o acréscimo da lidocaína à solução sangüínea hipercalêmica normotérmica.

Método: Foram estudados 26 cães, divididos em dois grupos de dez animais e um grupo de seis, de forma aleatória, conforme a solução cardioplégica de indução que receberam. O grupo I recebeu a solução composta por lidocaína $5 \mathrm{mg} / \mathrm{kg}$, cloreto de potássio $41,6 \mathrm{mEq} / \mathrm{l}$ em $180 \mathrm{ml}$ de sangue normotérmico. $O$ grupo II recebeu a solução anterior sem lidocaína e o grupo III recebeu somente os 180ml de sangue. A solução de manutenção foi a mesma para os 3 grupos composta por $120 \mathrm{ml}$ de sangue normotérmico, reinfundidos a cada 20min. Os animais foram submetidos à circulação extracorpórea, a duas horas de isquemia miocárdica e três horas de reperfusão. Foram avaliados mortalidade operatória, dosagens seriadas de troponina I, creatina quinase e lactado, débito cardíaco, fração de ejeção e fração de área das mitocôndrias. A análise estatística foi através dos testes exato de Fisher e a análise de variância de duplo fator complementada com o teste de Bonferroni.

Resultado: O grupo I apresentou menor mortalidade $(p=0,08)$, menor liberação da creatina quinase $(p<0,05)$ e menor comprometimento mitocondrial $(p=0,036)$. Não houve diferença entre os grupos quanto à liberação de troponina $I$, lactato e avaliação hemodinâmica.

Conclusão: Pode-se concluir que a lidocaína conferiu efeito protetor adicional ao miocárdio isquêmico e que os animais dos grupos I e II apresentaram alterações significativas, sugestivas de dano celular, com repercussões funcionais, para todos os parâmetros avaliados no decorrer do tempo.

Descritores: Proteção miocárdica. Solução cardioplégica. Lidocaína.

\section{Abstract}

Objective: The purpose of this research is to evaluate the efficacy of the lidocaine in myocardial protection with normothermic antegrade blood cardioplegia.

Method: Twenty six dogs were studied divided at random into two groups of ten and one group of six, depending on which cardioplegic solution they had received. Group I received a cardioplegic solution induction of lidocaine $5 \mathrm{mg} /$ $\mathrm{kg}, 41,6 \mathrm{mEq} / \mathrm{l}$ of $\mathrm{KCl}$ and $180 \mathrm{ml}$ of normothermic blood. Group II received the same solution except for the lidocaine and group III received only $180 \mathrm{ml}$ of blood. Every $20 \mathrm{~min} 120 \mathrm{ml}$ of normothermic blood was reinfused. All dogs underwent cardiopulmonary bypass, two hours of myocardial ischemia and three hours of reperfusion. These dogs were evaluated through operative mortality, myocardial enzymes such as cardiac troponin I and creatine kinase, lactate production, hemodynamic performance measured by ejection fraction and cardiac output, and morphometrics mitochondrial ultrastructural changes. Statistical analysis tests used to compare the results were the Fisher exact test and the twoway Anova.

Results: The results have shown that the animals from group $I$ in comparison to those of group II, had no mortality $(p=0,08)$, a lower production of creatine kinase $(p<0,05)$, lower mitochondrial ultrastructural changes $(p=0,036)$ and had no difference with cardiac troponin I production, lactate production and hemodynamic performance.

Conclusions: In conclusion it can be said that lidocaine offered an additional protection to the ischemic myocardial during cardiopulmonary bypass and that animals from groups I and II, with time, showed significant changes, compatible with cellular damage, for all the subjects evaluated.

Descriptors: Myocardial protection. Cardioplegic solution. Lidocaine.

Trabalho realizado no Instituto do Coração do Hospital das Clínicas da Faculdade de Medicina da Universidade de São Paulo. São Paulo, SP, Brasil.

Apresentado ao $29^{\circ}$ Congresso Nacional de Cirurgia Cardíaca.

Natal, RN, 4 a 6 de abril, 2002.

Endereço para correspondência: Ricardo Ribeiro Dias. Av. Dr.

Enéas de Carvalho

Aguiar, 44-2 ${ }^{\circ}$ andar.São Paulo, SP, Brasil. CEP 05403-000.

Tel.: (11) 3069-5318. e.mail: rdkz@uol.com.br 


\section{INTRODUÇÃO}

A lidocaína é fármaco anti-arrítmico classe I-B com capacidade de atuação direta na condutância transmembrana de cátions, principalmente do sódio, do potássio e do cálcio. Sua meia vida é de $100 \mathrm{~min}^{1}$.

A lidocaína apresenta capacidade de atuação direta no potencial de ação (PA) das fibras do sistema Hiss-Purkinje e dos miócitos, ou seja, atua diretamente sobre a atividade elétrica e mecânica do coração; através do bloqueio dos canais de sódio, bloqueando a fase zero do PA (ou fase da despolarização rápida) e através da lentificação da repolarização da membrana celular reduzindo a ascensão da despolarização normal da fase 4. Promove ainda a inibição do transporte de cálcio pela membrana mitocondrial, assim como sua liberação pelo retículo sarcoplasmático (etapa inicial do evento intracelular da contração muscular pelo processo actina-miosina) $)^{1,2}$.

Com o objetivo de se evitar a disfunção ventricular pósoperatória, diversas soluções cardioplégicas foram propostas $^{3,4}$, visando a proteção celular ${ }^{5}$ do miocárdio durante o período de isquemia intra-operatório.

Toda célula, quando submetida a um determinado estímulo, apresenta mecanismos específicos de adaptação, diretamente relacionados à natureza e intensidade do estímulo ${ }^{6}$. Conforme o período de isquemia, varia o grau e a reversibilidade da lesão celular imposta. Uma vez restabelecida a perfusão miocárdica, algumas vezes, apesar da manutenção da viabilidade celular do miócito, a recuperação da função contrátil e metabólica pode não ser imediata e nem completa ${ }^{7-10}$.

Afim de se evitar os fenômenos deletérios da agressão isquêmica durante a cirurgia cardiovascular, elaborou-se ensaio experimental com lidocaína, com o objetivo de avaliar a sua influência em solução cardioplégica sangüínea hipercalêmica normotérmica.

\section{MÉTODO}

Foram estudados 26 cães, com pesos que variaram entre 20 e $25 \mathrm{~kg}$, sem raças específicas, operados no período de fevereiro a novembro de 2000. Os animais foram obtidos no Biotério do Centro de Controle de Animais da Prefeitura do Município de São Paulo e manipulados em laboratório respeitando as diretrizes da National Institute of Health (USA) descritas no "Guide for the Care and Use of Laboratory Animals".

Esses animais foram divididos em 2 grupos de 10 e um terceiro grupo de 6 , conforme a solução cardioplégica de indução que receberam.

$\mathrm{O}$ grupo I recebeu a solução de indução $\mathrm{A}$, o grupo II, a solução B e o grupo III, a solução C.

A solução A foi composta por $180 \mathrm{ml}$ de sangue normotérmico, 7,5mEq de cloreto de potássio (concentração de $41,6 \mathrm{mEq} / \mathrm{l})$ e lidocaína na dose de $5 \mathrm{mg} / \mathrm{kg}$ do peso corpóreo do animal.

A solução B foi composta por $180 \mathrm{ml}$ de sangue normotérmico e 7,5mEq de cloreto de potássio (concentração de $41,6 \mathrm{mEq} / \mathrm{l})$.

A solução C constava de $180 \mathrm{ml}$ de sangue normotérmico.

A solução de manutenção foi semelhante para os 3 grupos e composta por $120 \mathrm{ml}$ de sangue normotérmico.

A distribuição das soluções cardioplégicas nos animais foi feita de forma aleatória.

\section{Procedimento Cirúrgico}

Os animais foram submetidos à anestesia geral com $30 \mathrm{a}$ $35 \mathrm{mg} / \mathrm{kg}$ de pentobarbital e $4 \mathrm{mg}$ de pancurônio.

Os cães foram submetidos à esternotomia mediana longitudinal, à CEC em normotermia, a duas horas de isquemia miocárdica, à infusão das soluções cardioplégicas $\mathrm{A}, \mathrm{B}$ ou $\mathrm{C}$, por 90seg e às reinfusões seriadas de sangue normotérmico a cada 20min, por 60seg. Após esse período de duas horas de isquemia, os animais foram observados durante as três horas de reperfusão que se seguiram, após as quais, foram sacrificados.

\section{Avaliação Intra-Operatória}

Realizada a partir da dosagem laboratorial da troponina I, da creatina quinase (CK) e do lactato, colhidos do seio coronário; da avaliação da função ventricular esquerda realizada através da ecocardiografia epicárdica e da aferição das medidas hemodinâmicas obtidas por cateter de Swan Ganz e do estudo quantitativo das mitocôndrias (morfometria) à microscopia eletrônica, em amostras colhidas do ápice do ventrículo esquerdo (figura 1).

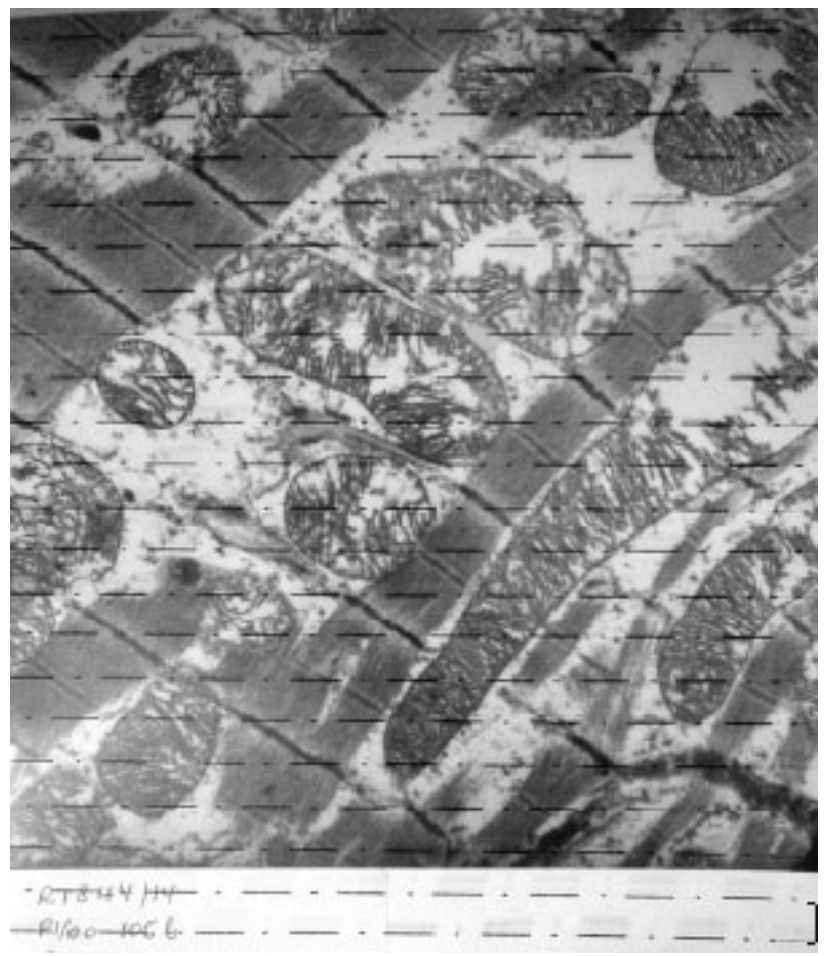

Fig. 1 - Imagem de uma micrografia eletrônica de um fragmento de miocárdio obtido do ápice do ventrículo esquerdo com aumento de 23.100 vezes, com a aplicação do sistema teste utilizado. 
As amostras foram colhidas nos tempos 1, 2, 3 e 4, sendo estes períodos respectivamente imediatamente anterior a isquemia miocárdica; após duas horas de isquemia; após uma hora e após três horas de reperfusão.

\section{Análise Estatística}

A existência de diferença entre os valores encontrados foi analisada através dos testes exato de Fisher para mortalidade operatória e análise de variância de duplo fator complementada com o teste de Bonferroni para as avaliações enzimáticas, metabólica, da função do ventrículo esquerdo e da morfometria das mitocôndrias.

\section{RESULTADOS}

Os animais do grupo I não apresentaram mortalidade, porém a diferença de resultados com o grupo II não atingiu significância estatística. No grupo III nenhum animal sobreviveu ao experimento (Tabela 1).

Tabela 1. Mortalidade operatória dos animais, em números absolutos e em porcentagem, para os grupos I, II e III, após serem submetidos em CEC, a duas horas de isquemia e três horas de reperfusão.

\begin{tabular}{lrr}
\hline & Mortalidade & Total \\
\hline Grupo I & 0 & $0 \%$ \\
Grupo II & 4 & $40 \%$ \\
Grupo III & 6 & $100 \%$ \\
\hline
\end{tabular}

Teste Estatístico - Teste Exato de Fisher Grupo I x Grupo II $\mathrm{p}=0,08$

A avaliação da variação enzimática através da dosagem seriada da troponina I demonstrou que não houve diferença significativa entre os grupos I e II até o tempo 3 (Tabela 2).

Tabela 2. Valores médios com seus respectivos desvios-padrão, da troponina I, expressos em ng/ml, nos animais dos grupos I, II e III, submetidos em CEC, a duas horas de isquemia e três horas de reperfusão.

\begin{tabular}{cccc}
\hline Troponina I & Grupo I & Grupo II & Grupo III \\
\hline Tempo 1 & $2,72 \pm 3,78$ & $1,33 \pm 1,04$ & $1,24 \pm 0,79$ \\
Tempo 2 & $2,5 \pm 2,37$ & $1,22 \pm 0,44$ & $2,73 \pm 1,77$ \\
Tempo 3 & $9,73 \pm 6,18$ & $13,56 \pm 9,61$ & $71,13 \pm 59,93$ \\
Tempo 4 & $17,44 \pm 8,87$ & $35,28 \pm 31,8$ & \\
\hline
\end{tabular}

Teste Estatístico - Análise de Variância de Duplo Fator

Grupo I x Grupo II - Tempo - p = 0,0001

Tratamento $-\mathrm{p}=0,7708$ - Tempo $1-\mathrm{p}>0,05$

Tempo $2-p>0,05-$ Tempo $3-p>0,05$

Porém em relação a CK houve uma tendência a diferença entre os grupos I e II, com menor liberação enzimática para o grupo I, sendo esta, significativa no tempo 3 (Tabela 3 ).
Tabela 3. Valores médios com seus respectivos desvios-padrão da CK, expressos em U/l, nos animais dos grupos I, II e III, submetidos em CEC, a duas horas de isquemia e três horas de reperfusão.

\begin{tabular}{crrr}
\hline CK & \multicolumn{1}{c}{ Grupo I } & \multicolumn{1}{c}{ Grupo II } & \multicolumn{1}{c}{ Grupo III } \\
\hline Tempo 1 & $137 \pm 79,87$ & $116 \pm 50,17$ & $113,5 \pm 30,55$ \\
Tempo 2 & $298,9 \pm 148,99$ & $359 \pm 105,92$ & $419 \pm 250,14$ \\
Tempo 3 & $538,7 \pm 192,72$ & $837,9 \pm 487,15$ & $2482,17 \pm 1330,1$ \\
Tempo 4 & $750,8 \pm 266,2$ & $933,8 \pm 330,87$ & \\
\hline
\end{tabular}

Teste Estatístico - Análise de Variância de Duplo Fator Grupo I x Grupo II - Tempo - p $<0,0001$

Tratamento $-\mathrm{p}=0,0661$ - Tempo $1-\mathrm{p}>0,05$

Tempo $2-\mathrm{p}>0,05-$ Tempo $3-\mathrm{p}<0,05$

A avaliação metabólica através da dosagem seriada do lactato (Tabela 4), a avaliação da função do ventrículo esquerdo, através das medidas do débito cardíaco com cateter de Swan Ganz (Tabela 5) e da quantificação da fração de ejeção pela ecocardiografia epicárdica (Tabela 6) não demonstrou diferença de comportamento entre os grupos I e II.

Tabela 4. Valores médios com seus respectivos desvios-padrão do lactato, expressos em mg/dl, nos animais dos grupos I, II e III, submetidos em CEC, a duas horas de isquemia e três horas de reperfusão.

\begin{tabular}{lccr}
\hline Lactato & Grupo I & Grupo II & \multicolumn{1}{c}{ Grupo III } \\
\hline Tempo 1 & $41,39 \pm 17,69$ & $37,69 \pm 16,22$ & $36,87 \pm 7,73$ \\
Tempo 2 & $90,72 \pm 21,47$ & $99,2 \pm 20,78$ & $108,5 \pm 28,71$ \\
Tempo 3 & $51,65 \pm 28,58$ & $51,81 \pm 77,82$ & $60,55 \pm 27,48$ \\
Tempo 4 & $57,25 \pm 38,03$ & $45,82 \pm 23,34$ & \\
\hline
\end{tabular}

Teste Estatístico - Análise de Variância de Duplo Fator Grupo I x Grupo II - Tempo - p $<0,0001$

Tratamento $-\mathrm{p}=0,8022$ - Tempo $1-\mathrm{p}>0,05$

Tempo $2-\mathrm{p}>0,05-$ Tempo $3-\mathrm{p}>0,05$

Tabela 5. Valores médios com seus respectivos desvios-padrão do débito cardíaco, expressos em 1/min, nos animais dos grupos I e II, submetidos em CEC, a duas horas de isquemia e três horas de reperfusão.

\begin{tabular}{ccc}
\hline Débito Cardíaco & Grupo I & Grupo II \\
\hline Tempo 1 & $1,95 \pm 0,73$ & $2,01 \pm 0,42$ \\
Tempo 3 & $1,15 \pm 0,39$ & $1,15 \pm 0,51$ \\
Tempo 4 & $0,88 \pm 0,36$ & $0,96 \pm 0,33$ \\
\hline
\end{tabular}

Teste Estatístico - Análise de Variância de Duplo Fator

Grupo I x Grupo II - Tempo - p = 0,0001

Tratamento $-\mathrm{p}=0,7069-$ Tempo $1-\mathrm{p}>0,05$

Tempo $2-\mathrm{p}>0,05-$ Tempo $3-\mathrm{p}>0,05$ 
Tabela 6. Valores médios com seus respectivos desvios-padrão da fração de ejeção nos animais dos grupos I e II, submetidos em CEC, a duas horas de isquemia e três horas de reperfusão.

\begin{tabular}{ccc}
\hline Fração de Ejeção & Grupo I & Grupo II \\
\hline Tempo 1 & $0,52 \pm 0,04$ & $0,6 \pm 0,08$ \\
Tempo 3 & $0,42 \pm 0,06$ & $0,35 \pm 0,13$ \\
Tempo 4 & $0,41 \pm 0,07$ & $0,38 \pm 0,14$ \\
\hline
\end{tabular}

Teste Estatístico - Análise de Variância de Duplo Fator

Grupo I x Grupo II - Tempo - p = 0,0001

Tratamento $-\mathrm{p}=0,6976-$ Tempo $1-\mathrm{p}>0,05$

Tempo $2-\mathrm{p}>0,05-$ Tempo $3-\mathrm{p}>0,05$

A avaliação morfométrica, obtida a partir do cálculo da fração de área das mitocôndrias dos fragmentos de miocárdio em análise, demonstrou que houve diferença significativa entre os grupos I e II, com menor aumento da fração de área das mitocôndrias no grupo I (Tabela 7).

Tabela 7. Valores médios com seus respectivos desvios-padrão das frações de áreas ocupadas por uma mitocôndria nas micrografias dos animais dos grupos I, II e III, submetidos em CEC, a duas horas de isquemia e três horas de reperfusão.

\begin{tabular}{cccc}
\hline Fração de Área & Grupo I & Grupo II & Grupo III \\
\hline Tempo 1 & $0,0064 \pm 0,0009$ & $0,0065 \pm 0,0012$ & $0,0090 \pm 0,0035$ \\
Tempo 2 & $0,0087 \pm 0,0015$ & $0,0106 \pm 0,0025$ & $0,0120 \pm 0,0028$ \\
Tempo 3 & $0,0061 \pm 0,0017$ & $0,0069 \pm 0,0018$ & $0,0076 \pm 0,0010$ \\
Tempo 4 & $0,0065 \pm 0,0014$ & $0,0074 \pm 0,0017$ & \\
\hline
\end{tabular}

Teste Estatístico - Análise de Variância de Duplo Fator

Grupo I x Grupo II - Tempo - p $<0,0001$

Tratamento $-\mathrm{p}=0,0365-$ Tempo $1-\mathrm{p}>0,05$

Tempo $2-\mathrm{p}<0,05$ - Tempo $3-\mathrm{p}>0,05$

Para todos os parâmetros analisados, nos três grupos, houve significativa alteração no decorrer do tempo.

Os resultados obtidos com o grupo III não foram estatisticamente analisados, servindo apenas como padrão de referência para comparação de dados com os grupos I e II, uma vez que todos os animais desse grupo morreram. Os resultados obtidos pelos grupos I e II, no tempo 4, não puderam ser submetidos à análise estatística comparativa, uma vez que $40 \%$ dos animais do grupo II morreram após a retirada da assistência mecânica da CEC (elevada perda de dados).

\section{COMENTÁRIOS}

A adequada proteção miocárdica com solução cardioplégica, segundo BUCKBERG ${ }^{11,12}$, só será conseguida, se esta promover a imediata parada cardíaca (em normotermia o coração com e sem atividade eletromecânica consome respectivamente de $6-9 \mathrm{mlO}_{2} \cdot 100 \mathrm{mg}^{-1} \cdot \mathrm{min}^{-1} \mathrm{e} 1 \mathrm{mlO}_{2} \cdot 100 \mathrm{mg}^{-1} \cdot \mathrm{min}^{-}$ ${ }^{1}$ ); com o resfriamento do miocárdio há redução do metabolismo cardíaco (à $20^{\circ} \mathrm{C}$ e à $10^{\circ} \mathrm{C}$ há consumo respectivo de $0,3 \mathrm{mlO}_{2} \cdot 100 \mathrm{mg}^{-1} \cdot \mathrm{min}^{-1}$ e $0,135 \mathrm{mlO}_{2} \cdot 100 \mathrm{mg}^{-1} \cdot \mathrm{min}^{-1}$ ) se for fornecido algum substrato energético ao miocárdio, para que possa haver continuada produção de energia, seja por via aeróbica ou anaeróbica; se a infusão de substâncias for capaz de manter o $\mathrm{pH}$ adequado para a otimização do metabolismo celular; e se for administrado algum estabilizador de membrana capaz de evitar o acúmulo intracelular de cálcio e sódio.

A lidocaína foi utilizada somente na solução de indução, pois uma vez que sua meia vida é de $100 \mathrm{~min}^{1}$, durante o período de $120 \mathrm{~min}$ a que os cães foram mantidos em isquemia, não seria necessária sua reinfusão.

A manutenção do animal em normotermia, assim como a infusão da solução cardioplégica normotérmica e a não infusão de qualquer outra substância durante as reinfusões com sangue, teve o objetivo de excluir a ação de outros mecanismos mioprotetores, além dos conferidos pela lidocaína. Dessa forma qualquer benefício adicional de proteção miocárdica pode ser atribuído à lidocaína, como já foi sugerido em trabalhos anteriores ${ }^{2,13-25}$ menor alteração da ultra-estrutura, menor consumo dos fosfatos de alta energia, menor incidência de arritmia ventricular de reperfusão, menor prejuízo da função hemodinâmica, menor alteração enzimática e até redução da área infartada.

No presente estudo, o período de anóxia dos animais submetidos à CEC foi deliberadamente extenso afim de que o sofrimento celular frente a agressão isquêmica imposta fosse inevitável, a menos que a influência da lidocaína fosse decisiva na preservação desse miocárdio agredido.

A mortalidade ocorrida nos grupos sem lidocaína demonstra a sua importância, apesar da ausência de diferença com significância estatística, entre os grupos I e II.

A variação enzimática apresentada para a troponina I e CK sugere menor dano celular nos miócitos dos animais do grupo I (a não utilização da CKMb, se deve ao fato do anticorpo anti-Mb ser espécie dependente e a sua utilização poderia implicar erros de interpretação).

As alterações da troponina I ocorridas no tempo 4, foram significativas, porém pela metodologia empregada, esse resultado não pode ser afirmado pela perda de $40 \%$ dos dados do grupo II. Em relação a CK, o grupo I apresentou menor elevação, com diferença significativa no tempo 3 .

A avaliação metabólica, a partir da dosagem seriada de lactato, demonstrou que, independentemente da solução cardioplégica recebida, a isquemia prolongada imposta ao miocárdio desses animais, resultou em elevada produção de lactato. Porém, sua produção aumentada, não implica necessariamente em prejuízo do desempenho hemodinâmico ${ }^{26}$, mas a intensidade da anaerobiose celular instalada, pode estar relacionada ao dano celular imposto pela isquemia, com prejuízo da viabilidade celular ${ }^{8,27}$ e conseqüente comprometimento da performance ventricular.

A lidocaína não foi capaz de diminuir a disfunção ventricular aguda determinada pela agressão isquêmica, apesar da ausência de mortalidade dos animais do grupo I. Resultados discordantes foram obtidos NASSER et al. ${ }^{19}$, LEE et al. ${ }^{20}$, LESNEFSKY et al. ${ }^{21}$ e SUNAMORI et al. ${ }^{24}$ que demonstram redução do dano isquêmico com melhora do 
desempenho ventricular com a infusão da lidocaína.

A avaliação da integridade celular obtida através da análise mitocondrial quantitativa visa a aferição da função metabólica e funcional do miócito. Foi através da morfometria que definitivamente a avaliação morfológica tornou-se objetiva e reprodutiva. GUNDERSEN et al. ${ }^{28}$ foram os responsáveis pela quantificação das formas e/ou estruturas, correlacionado-as a modelos matemáticos e não interpretativos, ou seja, correlacionaram parâmetros tridimensionais às medidas bidimensionais, possibilitando tratamento estatístico às observações morfológicas, desde que respeitados os critérios de seleção aleatória do material a ser analisado, e por isso, utilizado neste estudo.

A variação ascendente das frações de áreas das mitocôndrias, seja em relação à população total de mitocôndrias, seja em relação a uma mitocôndria, correspondeu ao momento da máxima agressão isquêmica a que os miócitos foram submetidos. Neste período, o grupo de cães que recebeu a lidocaína, apresentou a menor taxa de edema celular. A resposta celular foi imediata ao estímulo isquêmico e durante o período de reperfusão, além de não se prolongar, apresentou retorno a valores semelhantes aos iniciais. Se o maior edema celular está relacionado a pior proteção miocárdica ${ }^{14,15,18}$ o grupo que recebeu a lidocaína apresentou menor dano celular.

As mitocôndrias apresentam larguras que oscilam entre 0,5 e $1 \mathrm{~mm}$ e comprimentos de até $10 \mathrm{~mm}^{29}$. Suas áreas médias encontradas no início da CEC foram de três vezes o tamanho habitual (somente pela hemodiluição). Com o estímulo isquêmico, esse tamanho aumentou de forma variada, tendo sido maior, com significância estatística, no grupo em que o comprometimento celular foi maior, ou seja, no grupo II. O retorno das áreas a valores semelhantes aos iniciais, sugere que, pelo menos em relação às mitocôndrias, a lesão celular não foi irreversível.

\section{CONCLUSÕES}

A lidocaína, em associação a solução cardioplégica sangüínea hipercalêmica normotérmica, conferiu efeito protetor adicional ao miocárdio isquêmico durante a circulação extracorpórea.

Todos os animais dos grupos I e II, apresentaram alterações significativas, sugestivas de danos celulares, com repercussão funcional, para todos os parâmetros avaliados no decorrer do tempo.

\section{REFERÊNCIAS BIBLIOGRÁFICAS}

1. Bigger JR \& Hoffman BF - Drogas anti-arrítmicas. In: Goodman\&Gilman A, Rall TW, Nies AS, Taylor P, eds. As bases farmacológicas da terapêutica. 8. ed. Rio de Janeiro: Guanabara Koogan, 1991: 553-74.
2. Bengtsson L, Gunnes S, Norman B, Karlsson J - The effect of lidocaine on myocardial ischemia with asanguinous reperfusion: an in vitro study. Scand J Thorac Cardiovasc Surg 1991; 25: 141-6.

3. Demmy TL, Haggerty SP, Boley TM, Curtis JJ - Lack of cardioplegia uniformity in clinical myocardial preservation. Ann Thorac Surg 1994; 57: 649-51.

4. McGoon DC - The ongoing quest for ideal myocardial protection. A catalog of the recent English literature. J Thorac Cardiovasc Surg 1985; 89: 639-53.

5. Cordell AR - Milestones in the development of cardioplegia. Ann Thorac Surg 1995; 60: 793-6.

6. Robbins SL, Cotran RS, Kumar V - Lesão e adaptação celulares In: Robbins SL, Cotran RS, Kumar V, eds. Patologia estrutural e funcional. 3.ed. Rio de Janeiro: Interamericana, 1983: 1-37.

7. Jennings RB \& Reimer KA - Factors involved in salvaging ischemic myocardium: effect of reperfusion of arterial blood. Circulation 1993; 68(2 Pt 2): I25-36.

8. Ferrari R - Metabolic disturbances during myocardial ischemia and reperfusion. Am J Cardiol 1995; 76: 17B-24B.

9. Waxman K - Shock: ischemia, reperfusion, and inflammation. New Horiz 1996; 4: 153-60.

10. Hammond B \& Hess ML - The oxygen free radical system: potential mediator of myocardial injury. J Am Coll Cardiol 1985; 6: 215-20.

11. Buckberg GD - Strategies and logic of cardioplegic delivery to prevent, avoid, and reverse ischemic and reperfusion damage. J Thorac Cardiovasc Surg 1987; 93: 127-39.

12. Buckberg GD - A proposed "solution" to the cardioplegic controversy. J Thorac Cardiovasc Surg 1979; 77: 803-15.

13. Calafiore AM, Teodori G, Mezzetti A et al. - Intermittent antegrade warm blood cardioplegia. Ann Thorac Surg 1995; 59:398-402.

14. Sunamori M, Amano J, Okamura T, Suzuki A - Superior action of magnesium-lidocaine-l-aspartate cardioplegia to glucoseinsulin-potassium cardioplegia in experimental myocardial protection. Jpn J Surg 1982; 12: 372-80.

15. Kyo S, Laraia PJ, Magrassi P et al. - Myocardial protection by lidocaine during cardioplegia. J Surg Res 1983; 34: 533-42.

16. Fiore AC, Naunheim KS, Taub J et al. - Myocardial preservation using lidocaine blood cardioplegia. Ann Thorac Surg 1990; 50: 771-5. 
17. Baraka A, Hirt N, Dabbous A et al. - Lidocaine cardioplegia for prevention of reperfusion ventricular fibrillation. Ann Thorac Surg 1993; 55: 1529-33.

18. Okamura T, Sunamori M, Suzuki A - Protective effect of lidocaine in reperfused ischemic myocardium: evaluation by hemodynamic and biochemical study. Jpn Circ J 1982; 46: 657-62.

19. Nasser FN, Walls JT, Edwards WD, Harrison Jr. CE - Lidocaineinduced reduction in size of experimental myocardial infarction. Am J Cardiol 1980; 46: 967-75.

20. Lee R, Nitta T, Schmid RA, Schuessler RB, Harris KM, Gay Jr. WA - Retrograde infusion of lidocaine or L-arginine before reperfusion reduces myocardial infarct size. Ann Thorac Surg 1998; 65: 1353-9.

21. Lesnefsky EJ, VanBenthuysen KM, McMurtry IF, Shikes RH, Johnston Jr. RB, Horwitz LD - Lidocaine reduces canine infarct size and decreases release of a lipid peroxidation product. J Cardiovasc Pharmacol 1989; 13: 895-901.

22. Hearse DJ, O'Brien K, Braimbridge MV - Protection of the myocardium during ischemic arrest: dose-response curves for procaine and lignocaine in cardioplegic solutions. $\mathrm{J}$ Thorac Cardiovasc Surg 1981; 81: 873-9.
23. Sunamori M \& Harrison Jr. CE - Myocardial respiration and edema following hypothermic cardioplegia and anoxic arrest. J Thorac Cardiovasc Surg 1979; 78: 208-16.

24. Sunamori M, Okamura T, Amano J, Suma H, Suzuki A Myocardial protection by lidocaine hydrochloride in aortocoronary bypass surgery. Jpn J Surg 1982; 12: 93-7.

25. Rinne $\mathrm{T} \&$ Kaukinen $\mathrm{S}$ - Does lidocaine protect the heart during coronary revascularisation? Acta Anaesthesiol Scand 1998; 42: 936-40.

26. Carrier M, Tourigny A, Thoribé N et al. - Effects of cold and warm blood cardioplegia assessed by myocardial $\mathrm{pH}$ and release of metabolic markers. Ann Thorac Surg 1994; 58: 764-7.

27. Jennings RB \& Reimer KA - Lethal myocardial ischemic injury. Am J Pathol 1981; 102: 241-55.

28. Gundersen HJ, Bendtsen TF, Korbo Let al. - Some new, simple and efficient stereological methods and their use in pathological research and diagnosis. APMIS 1988; 96: 379-94.

29. Junqueira LC \& Carneiro J - A célula. In: Junqueira LC, Carneiro J, eds. Histologia Básica. 4. ed. Rio de Janeiro: Guanabara Koogan, 1985: 31-55. 